\title{
Optical clearing of skin enhanced with hyaluronic acid for increased contrast of optoacoustic imaging
}

\author{
Anton Liopo \\ Richard Su \\ Dmitri A. Tsyboulski \\ Alexander A. Oraevsky
}




\title{
Optical clearing of skin enhanced with hyaluronic acid for increased contrast of optoacoustic imaging
}

\author{
Anton Liopo, ${ }^{a}$ Richard Su, ${ }^{a, b}$ Dmitri A. Tsyboulski, ${ }^{a}$ and Alexander A. Oraevsky ${ }^{a, b, *}$ \\ ${ }^{a}$ TomoWave Laboratories, 6550 Mapleridge Street Suite 124, Houston, Texas 77081, United States \\ bUniversity of Houston, Department of Biomedical Engineering, 3600 Calhoun Road, Houston, Texas 77004, United States
}

\begin{abstract}
Enhanced delivery of optical clearing agents (OCA) through skin may improve sensitivity of optical and optoacoustic $(\mathrm{OA})$ methods of imaging, sensing, and monitoring. This report describes a two-step method for enhancement of light penetration through skin. Here, we demonstrate that topical application of hyaluronic acid (HA) improves skin penetration of hydrophilic and lipophilic OCA and thus enhances their performance. We examined the OC effect of $100 \%$ polyethylene and polypropylene glycols (PPGs) and their mixture after pretreatment by HA, and demonstrated significant increase in efficiency of light penetration through skin. Increased light transmission resulted in a significant increase of $O A$ image contrast in vitro. Topical pretreatment of skin for about 30 min with $0.5 \% \mathrm{HA}$ in aqueous solution offers effective delivery of low molecular weight OCA such as a mixture of PPG-425 and polyethylene glycol (PEG)-400. The developed approach of pretreatment by HA prior to application of clearing agents (PEG and PPG) resulted in a $\sim 47$-fold increase in transmission of red and near-infrared light and significantly enhanced contrast of OA images. $\odot 2016$ Society of Photo-Optical Instrumentation Engineers (SPIE) [DOI: 10.1117/1.JBO.21.8.081208]
\end{abstract}

Keywords: hyaluronic acid; optical clearing agents; skin; optoacoustic (photoacoustic) imaging.

Paper 160056SSRRR received Jan. 27, 2016; accepted for publication Apr. 18, 2016; published online May 27, 2016.

\section{Introduction}

Optical methods as a tool for clinical functional imaging of physiological conditions have been of great interest to the biomedical optics community. ${ }^{1-3}$ Over the past decade, noninvasive or minimally invasive methods of biomedical diagnostics and therapy utilizing spectroscopy and imaging techniques, including optoacoustic (OA) imaging, optical coherence tomography (OCT), confocal nonlinear microscopy and high speed imaging, in vivo fluorescent flow cytometry, and reflectance spectroscopy have seen widespread proliferation in biology and medicine. ${ }^{2,4-7}$ It is commonly understood that strong light scattering in skin tissue layers greatly diminishes light penetration through skin and limits the scope of these methods by reducing their contrast and penetration depth. ${ }^{2,8}$ By reducing this unwanted scattering, the performance of biomedical optical imaging techniques can be significantly improved.

Throughout published literature, one may find several hundred chemical compounds as potential skin penetration enhancers for different tasks, such as drug delivery, diagnostics, imaging biological objects, and visualization in vivo. ${ }^{9-17}$ For instance, the use of hyperosmotic biocompatible agents, such as glycerol, dimethylsulfoxide (DMSO), and polyethylene glycol (PEG) is well known to induce the effect of optical clearing (OC). ${ }^{18-22}$ One of the pioneering studies in OC demonstrated about a $50 \%$ increase in the optical transmittance of rat skin after a short (5 to $10 \mathrm{~min}$ ) administration of anhydrous glycerol. ${ }^{19}$ Recently, optical penetration depth and image contrast of photoacoustic microscopy enhanced by OC were measured as function of glycerol concentration. ${ }^{7}$ The maximum increase of the image brightness of $165 \%$ was achieved with

*Address all correspondence to: Alexander A. Oraevsky, E-mail: aao@ tomowave.com topical application of $60 \%$ glycerol in water. OC of biological tissues may be achieved utilizing two main mechanisms-tissue dehydration and refractive index matching. ${ }^{22}$ Most of the previously reported methods of optical clearance utilized tissue dehydration induced by the optical clearing agents (OCA) interacting with external layers of tissue..$^{3,14,22-28}$ Khan et al. ${ }^{29}$ used a PPG and PEG mixture to reduce dermal optical scattering and increase penetration depth of OCT by $17 \%$ in human skin in vivo. Tuchin ${ }^{30}$ reviewed available methods of tissue OC in various tissue structures.

Exogenous hyperosmotic chemicals used as OCAs penetrate into tissues and displace water, thereby reducing refractive index mismatch between scatterers, such as collagen fibrils, cell organelles, and interstitial fluids or cytoplasm. ${ }^{14,27}$ The OCA list includes well-known chemical compounds, e.g., glucose, glycerol, dextrose, fructose, sucrose, sorbitol, xylitol, propylene and polypropylene glycol (PPG), PEG, butylene glycol, DMSO, ethanol, oleic acid, sodium lauryl sulfate, azone, and thiazone. ${ }^{3,14}$ Typically, OCAs can be classified as being part of three main groups-sugars, alcohols, and electrolyte solutions. ${ }^{27}$ Some agents, such as DMSO and PEG, can be used both as OCAs and clearing enhancers, or used together with chemical or physical enhancers to increase diffusion across stratum corneum and other skin layers. ${ }^{14,23,26}$ Various methods for enhancing penetration of OCA into tissue, such as physical methods, chemical-penetration enhancers, and combinations of physical and chemical methods, can be utilized. ${ }^{3}$

As presented in the lower part of Fig. 1, molecular agents may undergo three potential pathways when penetrating skin. The first is the intercellular route through the stratum corneum, i.e., through the structured intercellular lipid bilayer. ${ }^{31}$ This

$1083-3668 / 2016 / \$ 25.00$ @ 2016 SPIE 


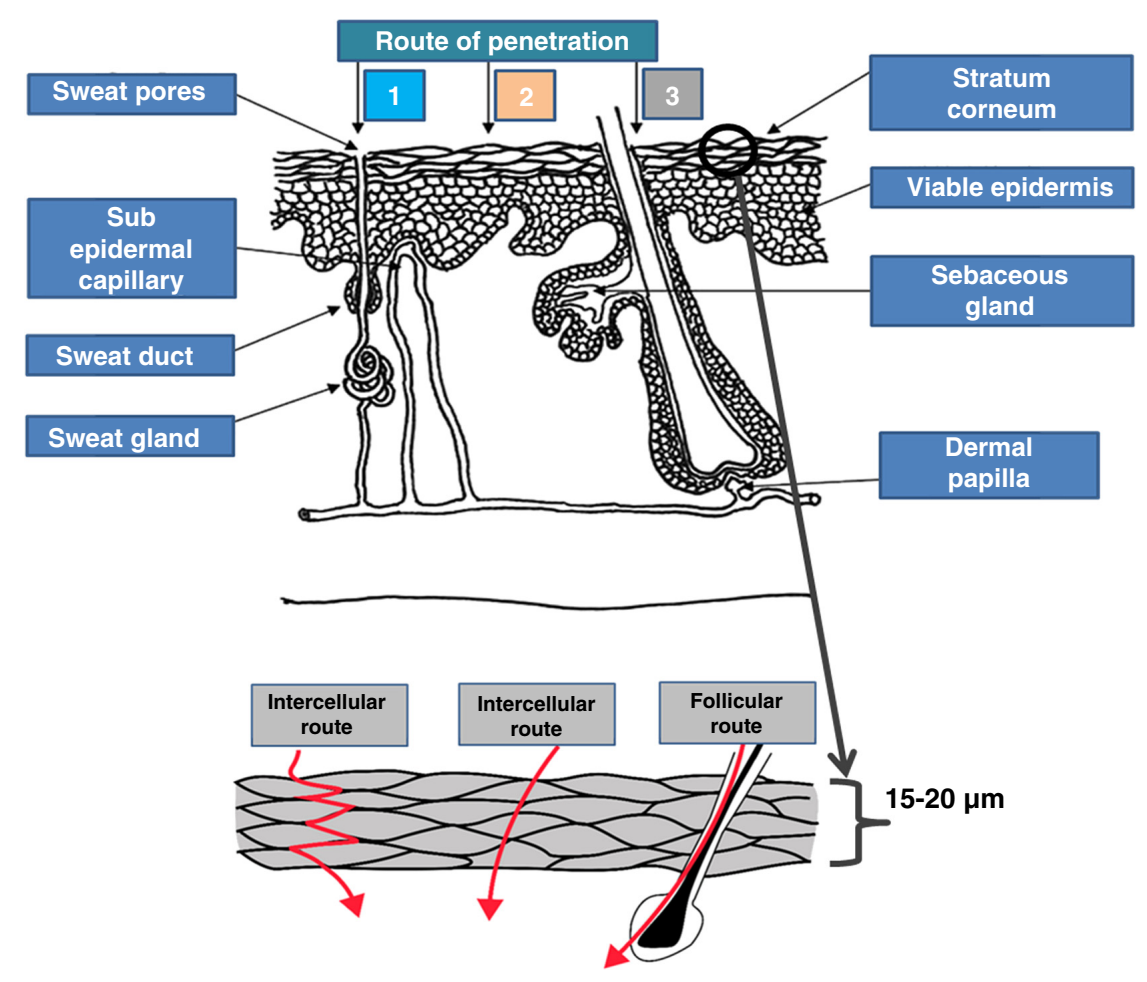

Fig. 1 A diagram showing routes of skin penetration by OCA, adopted from Ref. 11: (1) through the sweat ducts; (2) directly across the stratum corneum; and (3) via the hair follicles. Permeation routes directly through the stratum corneum include intercellular diffusion through the lipid lamellae, intracellular or transcellular diffusion through both the keratinocytes and lipid lamellae, and diffusion through appendages, i.e., hair follicles and sweat ducts.

transport process involves sequential diffusion and partitioning between the polar head group regions and the long alkyl chains of the lipids. ${ }^{32}$ The second is the transcellular route, which can be utilized by small hydrophilic substances. ${ }^{11}$ The third minor route is through hair follicles, associated sebaceous glands, and sweat ducts.

Typically, the OC process takes more than an hour, ${ }^{33}$ but it can be accelerated with chemical or physical enhancers. ${ }^{3}$ We propose hyaluronic acid (HA) as a potential chemical enhancer because of its ability to change physiological properties of skin (stratum corneum) through water redistribution. Hyaluronan or HA is a nonsulphated, straight chain glycosaminoglycan polymer of the extracellular matrix. It can reach several thousands of sugar units (carbohydrates) in length. ${ }^{34}$ The biological functions of HA include maintenance of the elastoviscosity of liquid connective tissues, control of tissue hydration and water transport, and numerous receptor-mediated roles in cells. ${ }^{35,36}$

The structure of the disaccharide is energetically very stable. ${ }^{37} \mathrm{HA}$ is highly nonantigenic and nonimmunogenic, and has poor interaction with blood components. ${ }^{38,39}$ The unique viscoelastic nature of HA along with its biocompatibility has led to its use in a number of clinical applications, including the supplementation of joint fluid in arthritis, in eye surgery, and enhancement of healing and regeneration of surgical wounds. ${ }^{40-43}$ HA has been investigated for delivery of different agents for various administration routes, including ophthalmic, nasal, pulmonary, parenteral and topical drugs, and gene delivery. ${ }^{42,44-46}$ Dermatological and cosmetic applications using of HA have been extensively probed. ${ }^{47} \mathrm{HA}$-based formulations may also be capable of protecting the skin against ultraviolet irradiation due to the free radical scavenging properties of
HA. ${ }^{48}$ Radio-labeled HA was found not only to penetrate the skin of nude mice and humans, but also to aid the transport of diclofenac to the epidermis. ${ }^{49}$ The results ${ }^{38}$ show that HA is not cytotoxic and shows good biocompatibility. Studies ${ }^{38,49}$ collectively imply that HA's influence on the local delivery of chemical compounds to the skin depends upon the skin characteristics and species of skin used, with the compounds depot forming in deeper layers of mouse skin (dermis) compared with humans (epidermis).

HA hydrogels and nanocomposites were used in different experimental and preclinical models. ${ }^{34,50,51}$ Amphiphilic HA derivatives bearing hydrophobic indocyanine green dye and hydrophilic PEG were synthesized through the use of condensation and copper-catalyzed click cyclization reactions. ${ }^{52}$ The repair effect of HA and vaseline was demonstrated in dermal blood vessel study for peripheral vascular diseases. ${ }^{53}$ Two kinds of fluorescent $\mathrm{HA}$ analogs, one serving as a normal imaging agent and the other used as a biosensitive contrast agent, were developed for the investigation of HA uptake and degradation. ${ }^{54}$ An increase in HA synthesis, cellular uptake, and metabolism occurs during the remodeling of tissue microenvironments following injury. ${ }^{55}$ In recent years, HA has emerged as a promising candidate for intracellular delivery of various therapeutic and imaging agents because of its innate ability to recognize specific cellular receptors. ${ }^{56}$ Injectable hydrogels based on HA and PEG were designed as biodegradable matrices for cartilage tissue engineering. ${ }^{57}$ It should be noted that hydrogels are not OCA, and in fact they can increase optical scattering of tissues. ${ }^{58}$

In this report, we demonstrate the use of aqueous solutions of HA without modification and functionalization as potential 
enhancers for delivery of OCAs, such as PPG and PEG, and their mixtures, through pig skin. The aim of the present study is to demonstrate OC of skin after using chemical pretreatment with HA.

\section{Materials and Methods}

\subsection{Reagents}

PPG average $M_{\mathrm{n}} 425$ (PPG-425), PEG average $M_{\mathrm{n}} 400$ (PEG$400)$, nickel(II) sulfate hexahydrate $\left(\mathrm{NiSO}_{4}\right)$, gelatin from porcine skin, strength 300, HA, and phosphate buffered saline (PBS) were purchased from Sigma-Aldrich. Ultrapure water $(18.2 \mathrm{M} \Omega \times \mathrm{cm}$ at $25^{\circ} \mathrm{C}$ ) was used throughout the work. Hexadecyl-trimethylammonium bromide (CTAB), silver nitrate, potassium carbonate $\left(\mathrm{K}_{2} \mathrm{CO}_{3}\right)$, gold(III) chloride trihydrate $\left(\mathrm{HAuCl}_{4}\right)$, sodium borohydride $\left(\mathrm{NaBH}_{4}\right)$, silver nitrate $\left(\mathrm{AgNO}_{3}\right)$, hydrochloric acid $(\mathrm{HCl})$, and hydroquinone were purchased from Sigma-Aldrich, methoxy PEG thiol, MW 5000 (mPEG-Thiol or PEG, Laysan Bio Inc.). Synthesized gold nanorods (GNR) and $\mathrm{NiSO}_{4}$ solutions were used as contrast agents for OA imaging.

\subsection{Gold Nanorods}

GNR were synthesized with the hydroquinone seedless method and used as contrast agents for OA, according to the protocol of $\mathrm{Xu}$ et al. ${ }^{59}$ with our small modification. ${ }^{60}$ For the synthesis of GNR, both modifications of seedless hydroquinone initialized protocols were applied. GNR were prepared by mixing CTAB $(5.00 \mathrm{~mL}, 0.1 \mathrm{M}), \mathrm{HAuCl}_{4}(5.00 \mathrm{mM}, 1.0 \mathrm{mM}), \mathrm{AgNO}_{3}$ $(50 \mu \mathrm{L}, 100 \mathrm{mM})$, and hydroquinone $(250 \mu \mathrm{L}, 100 \mathrm{mM})$. After that, the reaction was started by adding $15 \mu \mathrm{L}$ freshly prepared $0.01 \mathrm{M} \mathrm{NaBH}_{4}$ solution. The mixed solution was stirred for a short time and left undisturbed at $28^{\circ} \mathrm{C}$ for growth of GNR overnight. This protocol is for $757 \mathrm{~nm} .{ }^{59,60} \mathrm{GNR}$ were prepared by mixing CTAB $(5.00 \mathrm{~mL}, 0.1 \mathrm{M}), \mathrm{HAuCl}_{4}(5.00 \mathrm{mM}$, $1.0 \mathrm{mM}), \mathrm{AgNO}_{3}(50 \mu \mathrm{L}, 100 \mathrm{mM})$, hydroquinone $(500 \mu \mathrm{L}$, $50 \mathrm{mM})$, and $20 \mu \mathrm{L} \mathrm{HCl}(1.19 \mathrm{M})$. After equilibration, the reaction was started by adding $15 \mu \mathrm{L}$ freshly prepared $0.01 \mathrm{M} \mathrm{NaBH}_{4}$ solution. This protocol is for $1064 \mathrm{~nm}^{59,60}$ Previously, we described general strategy for stabilization of GNR with thiol-terminal PEG, which displaces the original bilayer of surfactant CTAB to provide reduced potential connection of GNR with the tube internal wall. ${ }^{61,62}$ As a result, we got two types of pegylated GNR with different maxima of localized surface plasmon resonance (LSPR): one at the wavelength of $757 \mathrm{~nm}$ and the other at $1064 \mathrm{~nm}$, which correspond to the two short-pulse lasers, the Alexandrite laser $(757 \mathrm{~nm})$, and the Nd:YAG laser $(1064 \mathrm{~nm})$.

\subsection{Skin}

The skin was harvested from the belly of young pig $(<1$ years old), and the underlying muscle and fat layers were removed carefully. Pieces of porcine skin were purchased from a food store, making these samples exempt from National Institutes of Health guidelines and regulations regarding animal studies according to the Institutional Animal Care and Use Committee. Nonfrozen, freshly prepared $1.30 \pm 0.14-\mathrm{mm}$ thick skin segments $(\sim 1.5 \times 1.5 \mathrm{~cm})$ were exposed to clearing agents for different periods of time, up to $120 \mathrm{~min}$, and placed over a resolution target. Images of these samples before and after exposure were recorded with a high-resolution digital camera. For three-dimensional (3-D) OA imaging, we used rectangular pieces $(\sim 25 \times 75 \mathrm{~mm})$. Skin was wrapped around four garolite rods, creating a rectangular shape with tubes with GNR, and nickel sulfate was inside the garolite rod frame.

\subsection{Optical Property Skin and Contrast Agents}

Optical measurements were performed using pig skin samples in vitro. Transmission spectra were acquired from the pig skin samples with an Ocean Optics USB4000 spectrophotometer with an acquisition band between 500 and $1400 \mathrm{~nm}$. The light source used was a Micropack Halogen lamp-model HL-2000 FHSA. The control for spectral properties of GNR (UV-VIS spectra in the range of 400 to $1100 \mathrm{~nm}$ ) was done using Beckman 530 and Thermo Scientific Evolution 201 spectrophotometers. Optical absorption spectra in the wavelength range longer than $1100 \mathrm{~nm}$ were obtained on a Cary 5000 (Varian) UV-VIS-NIR spectrophotometer.

\subsection{Optoacoustic Imaging System}

Imaging experiments reported here were made with a commercial 3-D laser optoacoustic imaging system (LOIS-3-D) developed for preclinical research by TomoWave Laboratories (Houston, Texas) and introduced in our earlier publications. ${ }^{4,62-65}$ Laser output consisted of Alexandrite laser (757 nm, $50 \mathrm{~ns} /$ pulse) and Nd:YAG (1064 nm, $9 \mathrm{~ns} /$ pulse), which was manufactured as a single unit with coaxial output beams (Quanta Systems, Solbiate Olona, Italy). ${ }^{62}$ OA tomography was performed using the imaging module of LOIS-3-D, as shown in Fig. 2. The stainless steel tank holds a 115-deg arc-shaped array of 96 wide-band ultrasonic transducers connected to our custom-designed electronics and our quad fiberoptic bundle, which was proven to be a viable light delivery system for small animal imaging (see Fig. 2). ${ }^{62,63}$ The pulsed laser energy was about $6.5 \mathrm{~mJ}$ out of each of the four fiberoptic illuminators, which corresponded to the total effective optical fluence of $2.5 \mathrm{~mJ} / \mathrm{cm}^{2}$. The system has low noise equivalent pressure of $2 \mathrm{~Pa}$ without averaging, ${ }^{64}$ which permits reliable visualization of the OA contrast generated by variation of the tissue optical absorption of $0.12 \mathrm{~cm}^{-1}$ with 4-pulse averaging, making the total image acquisition time 2 min. 65

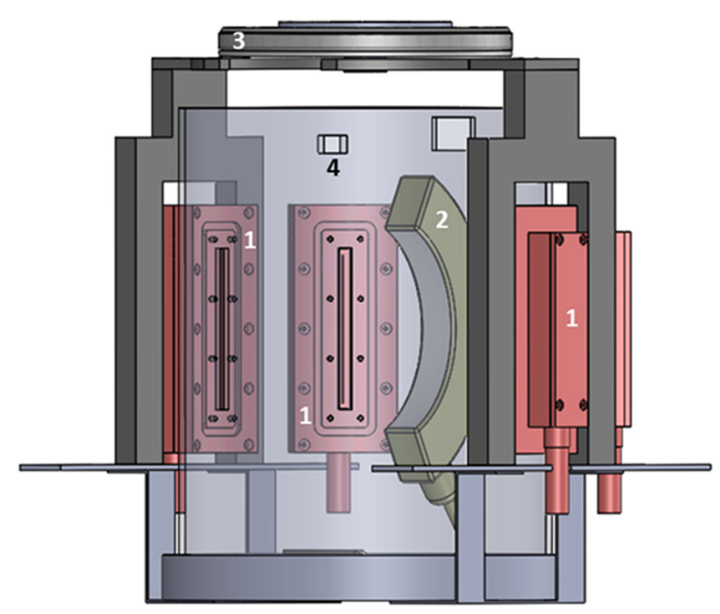

Fig. 2 The imaging module of the laser OA imaging system, LOIS-3-D (TomoWave Laboratories, Houston, Texas), which includes (1) quad fiberoptic bundle of the light delivery system, (2) arc array of ultrasonic transducers, (3) computer controlled rotational stage, and (4) video camera. 
The phantom consisted of four garolite rods for support, angled target tubes (tubes with GNR and nickel sulfate), and a layer of skin of $2.5 \mathrm{~cm}$ height creating a rectangular shell centered around the section of the tubes, where the focus of the arc would be (see Fig. 3). A custom-made bracket supported two polytetrafluoroethylene (PTFE) tubes with internal diameter $510 \mu \mathrm{m}$ and wall thickness of $150 \mathrm{~mm}$. One tube was filled with GNR nanoparticle suspension and the other was filled with nickel sulfate solution in water. Nickel sulfate was used with the same optical density as GNR to enable quantitative measurements of changes in the $\mathrm{OA}$ image brightness of the tubes covered with pig skin that occur after OC of the skin. Figure 3 shows a picture of the phantom prepared for imaging.

OA contrast of GNR and nickel sulfate was compared for images obtained before and after application of $0.5 \%$ solution of HA (pretreatment) and low molecular weight $100 \%$ PPG425 or $100 \%$ PEG-400 and PPG-PEG mixture $1: 1$. Imaging experiments were repeated three times. Signal processing was similar to that described in our previous publications using deconvolution of transducer impulse response and a bandpass filter. ${ }^{66}$ Image reconstruction was made with a filtered back projection algorithm utilizing a half-time data set. ${ }^{67} 3-\mathrm{D}$ visualization was done with LOIS-View, a software package developed at TomoWave based on the open software development kit source developed by 3-D slicer. ${ }^{68}$ We created a segmentation module of LOIS-3-D software that allowed one to draw regions of interest along the axial slices of the reconstructed scan, interpolate, and connect them. This segmented region was statistically analyzed by measuring mean and median brightness values and their standard deviation. Each tube was segmented into a region of interest that was a cylinder of $0.6 \mathrm{~mm}$ in diameter and height of $5 \mathrm{~mm}$. The average brightness of OA images was measured in the central area of the images illuminated more evenly than the adjacent areas. The image processing parameters such as scalar opacity, color mapping, and gradient opacity were held constant between all objects and scans such that one can directly compare the image brightness across all images in a qualitative manner and assign their colors using a linear color palette. From lowest to highest, OA contrast is black to red, with voxels deemed noise colored black (transparent).

\section{Results}

First, we demonstrate the OC effect after application of clearing agents without HA. Figure 4 shows consecutive photographs of skin samples treated with PPG and PEG solutions for 10, 20, 45, 90 , and $120 \mathrm{~min}$. We observed significant OC in these samples after $\sim 90$ and $120 \mathrm{~min}$ of exposure to PPG and PEG mixtures,

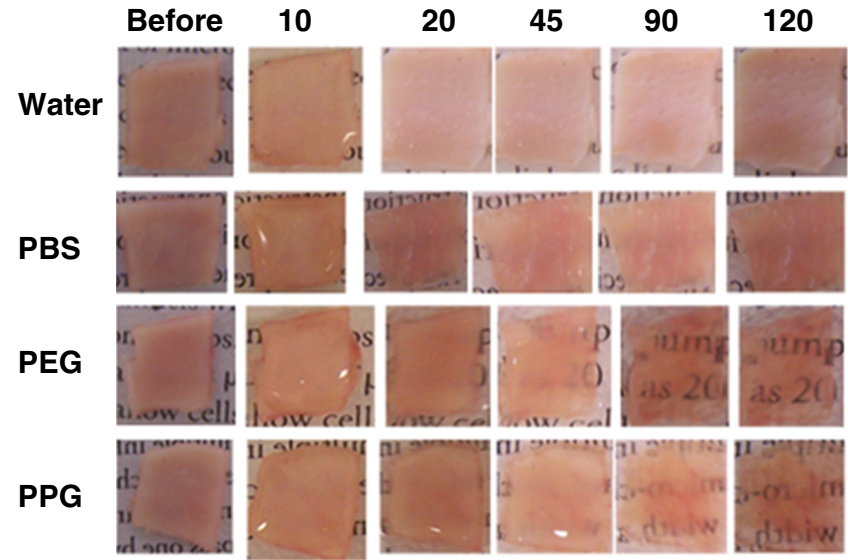

Fig. 4 Dynamics of OC after treatment of pig skin with control (water and PBS) and active (PEG and PPG) clearing agents. Time values are in minutes.

respectively. In the course of experiments, it was discovered that when the same clearing agents were used in combination with HA pretreatment, a similar clearing effect was achieved in a significantly shorter period of time.

Figure 5 shows the comparison of measured unscattered transmission spectra from skin samples after clearing procedures with and without HA pretreatment. The recorded spectra were not normalized by the spectral profile of the illumination source. We observed that application of only PPG, PEG, and their mixture for $60 \mathrm{~min}$ led to a relatively small clearing effect [Fig. 5(a)]. With HA pretreatment for $30 \mathrm{~min}$, followed by treatment with PPG, PEG, and their mixture for 15 and $45 \mathrm{~min}$ [Figs. 5(b) and 5(c)], OC was significantly improved. The experiment showed that pretreatment with HA enhanced OC of skin even after a relatively short PPG-PEG mixture application. After $45 \mathrm{~min}$, the transmission of unscattered light through skin samples increased at least eight times.

To quantify increase in the skin transmission after OC, we compared the transmission spectra of cleared skin samples shown in Fig. 5(c) relative to a control, which was measured from a sample pretreated with HA solution for $30 \mathrm{~min}$ and then treated by an inactive PBS solution for $45 \mathrm{~min}$. Figure 6 shows photographs of the skin samples before and after the clearing procedure (a) and the normalized transmission spectra (b). This figure demonstrates a $\sim 47$-fold increase of the optical fluence transmitted through skin. To demonstrate potential benefits of skin clearing, we performed a series of OA imaging experiments of a test phantom presented.

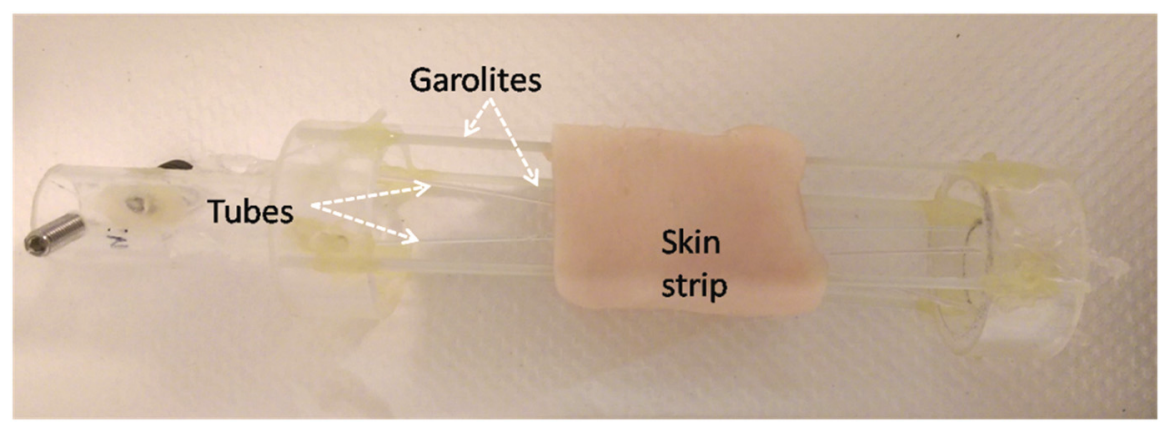

Fig. 3 Preparation of pig skin sample for OA scanning with LOIS-3-D. Skin is wrapped around a holder containing PTFE tubes filled with contrast agents. 


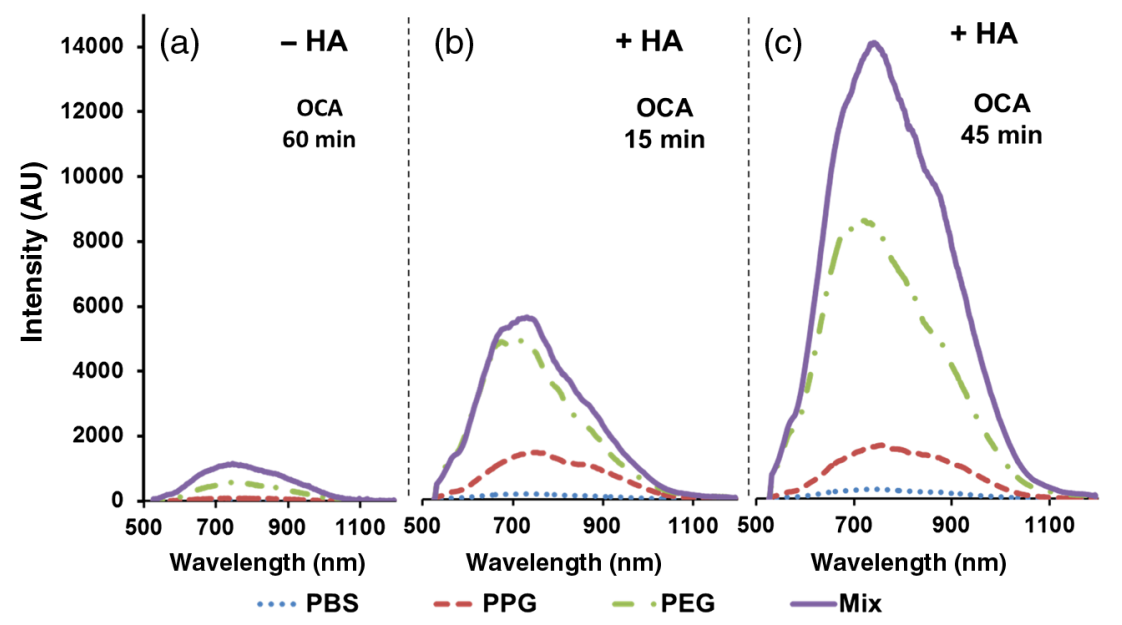

Fig. 5 Unscattered light transmission spectra through skin samples measured after different OC procedures: (a) without HA pretreatment, using only PPG, PEG, and their mixture as clearing agents for $60 \mathrm{~min}$, and (b, c) with HA pretreatment for $30 \mathrm{~min}$, followed by treatment with the same agents for 15 and $45 \mathrm{~min}$, respectively.

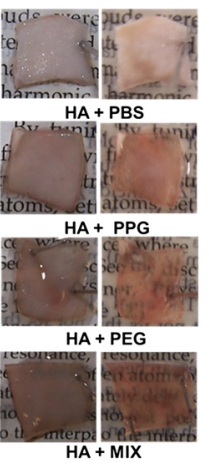

(a)

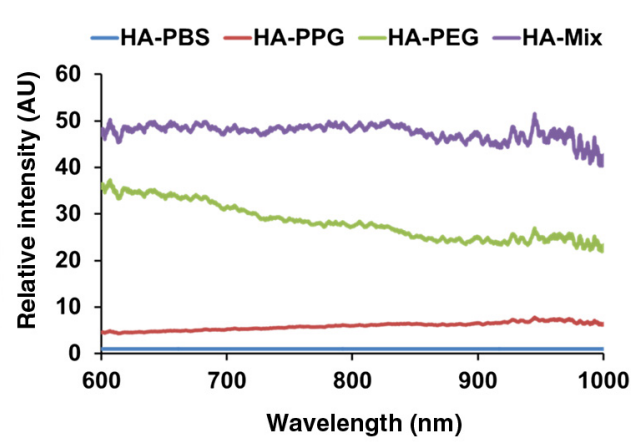

(b)
Fig. 6 (a) Photographs of pig skin samples before and after OC procedure consisting of 30-min HA pretreatment and 45-min treatment with PBS control, PPG, PEG, and their mixture. (b) Normalized unscattered light transmission spectra after $\mathrm{OC}$ procedures. The spectrum of unscattered light transmitted through HA-PBS treated skin was used as a reference.

Both two-component GNR and nickel sulfate solutions were used as optical contrasts in these experiments. Absorption spectra of pegylated GNR with maximum LSPR at 757 and $1064 \mathrm{~nm}$ are shown in Fig. 7. By adjusting concentrations of these components in a mixture, it is possible to tune the absorption coefficients at these wavelengths to desired values.

Figure 8 shows the absorption spectra of a two-component GNR solution matching absorbance of the nickel sulfate solution at both 757- and 1064-nm laser excitation wavelengths.

Figure 9 shows a photograph of the phantom positioned for scanning (a) and the reconstructed images of the test tubes (b). Skin in the test phantom was subject to the same OC procedure that begins with 30-min pretreatment with HA and is followed by 45 -min treatment with the PEG-PPG mixture. OA images of the phantom were acquired before and after the OC procedure. Projections of 3-D volumes represent segmented portions of tubes filled with GNR and $\mathrm{NiSO}_{4}$, which were reconstructed after scanning with 757- and 1064-nm lasers Fig. 9(b). Figure 9 shows a photograph of the phantom and the ultrasound probe positioned for scanning (a), and the reconstructed images of test tubes filled with contrast agents before and after the clearing procedure (b).

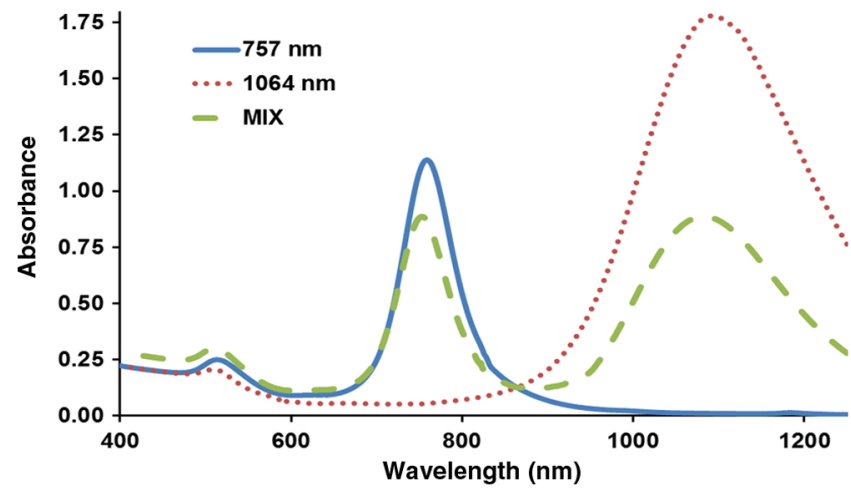

Fig. 7 UV-VIS-NIR spectra of pegylated gold nanorods with maximum LSPR at $757 \mathrm{~nm}$ (Alexandrite laser) and $1064 \mathrm{~nm}$ (Nd:YAG laser) and their mixture adjusted for the same optical density for both wavelengths. The mixture solution demonstrated LSPR peaks very similar to each GNR measured separately.

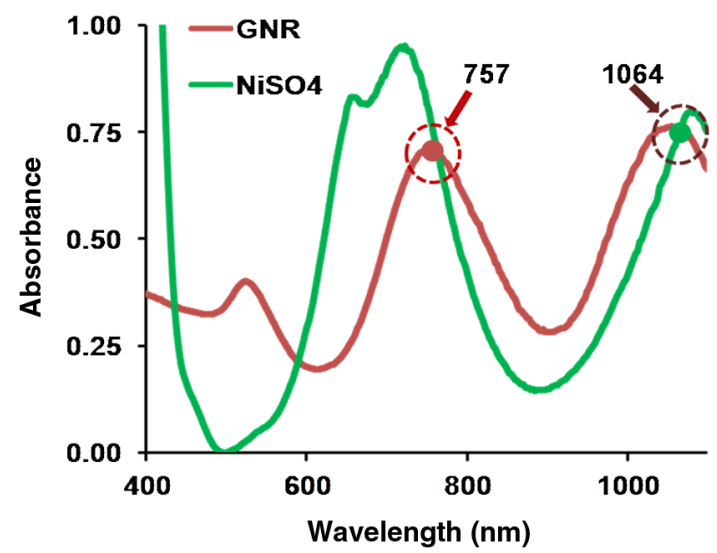

Fig. 8 Absorption spectra of a two-component GNR solution and nickel sulfate solution with matched absorbance at 757 and $1064 \mathrm{~nm}$.

We found that application of the OC procedure proposed in this study resulted in significant enhancement in the average pixel brightness for both contrast agents and for both wavelengths. 
(a)

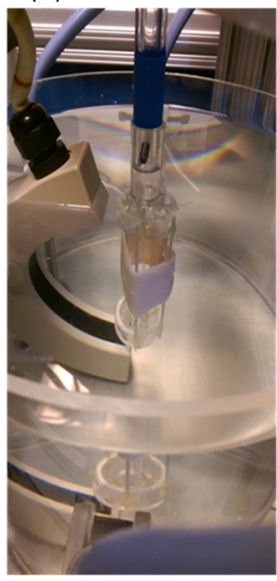

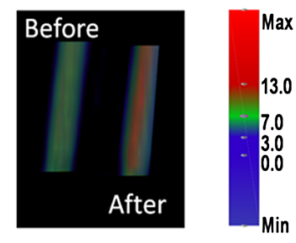

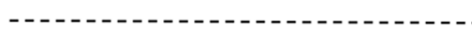

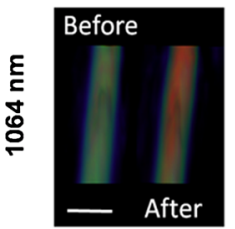

$\mathrm{NiSO}_{4} \quad 0.6 \mathrm{M}$

(b)

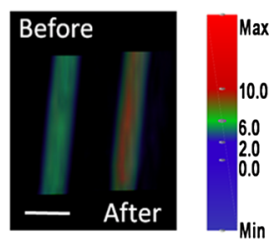

Fig. 9 (a) Photograph of the experimental setup showing the arcshaped ultrasonic probe and phantom in their vertical orientation during OA scanning. (b) 3-D image projections of a segmented portion of GNR and $\mathrm{NiSO}_{4}$ tubes reconstructed after OA scanning with 757 and $1064 \mathrm{~nm}$. A significant contrast increase can be seen after pretreatment with $\mathrm{HA}$ as opposed to the image obtained before the treatment.

\section{Discussion}

PEG and PPG are chemicals that fall into the broad chemical classification known as "alcohols" as any organic chemical that contains an $-\mathrm{OH}$ (oxygen/hydrogen) functional group. The US Food and Drug Administration (FDA) approved PEGs for medical applications. Propylene glycol is among the most thoroughly tested and well understood chemicals. It has been affirmed by the FDA as a "generally recognized as safe" compound and is permitted for use in food and drug products. FDA permits use of the term "alcohol-free" for cosmetics that contain alcohols other than ethanol. HA is approved by the FDA for many different clinical applications from dermal fillers to injectable substations and viscosupplements.

The disaccharide subunit of HA consists of D-glucuronic acid and D-N-acetyl-glucosamine linked together. Both the glucuronic acid and amino sugar are related to the glucose family. Glucose in its beta configuration allows all of its bulky groups to be in sterically favorable equatorial positions, while all of the small hydrogen atoms occupy the less sterically favorable axial positions. Thus, the structure of the disaccharide is energetically very stable. ${ }^{35,36} \mathrm{HA}$ is one of the most hydrophilic molecules in nature and can be described as natural moisturizer. As mentioned above, the HA polymer in solution assumes a stiffened helical configuration that can be attributed to hydrogen bonding between the hydroxyl groups along the chain. As a result, a coil structure is formed that traps water $\sim 1000$ times higher than its own weight. ${ }^{34} \mathrm{HA}$ can be obtained by self-assembling of amphiphilic graft copolymers with different polymer molecules, such as polylactic acid, poly-lactide-coglycolic acid chains of PEG and PPG, and others. ${ }^{69-71}$ The amphiphilic properties of HA are base for both hydrophilic and lipophilic pathways of penetration into skin. The amphiphilic HA derivatives dissolved in water form self-assemblies in which the near-infrared dyes were tightly packed and arranged to form dimers or H-aggregates. By utilizing HA derivatives as contrast agents, tumors were clearly visualized by optical imaging as well as by OA tomography. ${ }^{52}$

Because skin is composed of alternating lipophilic and hydrophilic layers, amphipathic compounds that possess both properties may be absorbed by the skin more effectively. Hydration is the most widely used and safest method to increase skin penetration for both hydrophilic and lipophilic permeants. Ideally, amphipathic compounds should minimize the lipophilic-hydrophilic interface to overcome physiological forces in the skin. Studies ${ }^{19,72}$ have demonstrated that the injection of hyperosmotic agents into rat dermis could significantly reduce light scattering. However, penetration of glycerol and PEG through intact skin is very minimal and extremely slow because these agents are hydrophilic and penetrate the lipophilic stratum corneum poorly. At the same time, it has been reported $^{29,33}$ that OC potential was significantly higher for the lower molecular weight combined PPG- and PEG-based prepolymer mixture as compared to either glycerol or prepolymer alone.

Lipophilic PPG- and hydrophilic PEG-based polymers have a refraction index of 1.47 , which is close to that of dermal collagen. ${ }^{29}$ The OC synergistic effect was shown when all three components, OCA PEG-400 and the two chemical-penetration enhancers thiazone and 1,2-propanediol, were applied together with physical massage. ${ }^{73}$ These results were confirmed by application of low molecular weight PEG-400 in vivo. ${ }^{73}$ Our data demonstrate a synergistic effect of OC when an HA, PEG400, and PPG-425 mixture was used. The unscattered transmission of light through pig skin is significantly higher for the combined PPG- and PEG-based prepolymer mixture than PPG and PEG alone [Fig. 5(a)]. This effect is significantly enhanced if pretreatment of skin with HA was made before application of the polymers [Figs. 5(b) and 5(c)]. The combined effect of the PPG- and PEG-based mixture on light penetration through skin was also evaluated in OA images (Fig. 9). As we found experimentally, ${ }^{65}$ water suspension of GNR with aspect ratio (AR) 3.3 has LSPR around $757 \mathrm{~nm}$, and GNR with AR near 6.0 have LSPR around 1064 and concentrations of GNR-757 and GNR-1064 250 and 150 pM for optical density 1.0, respectively. In the current experiments we used GNR concentration of $400 \mathrm{pM}$ for both wavelengths, which corresponds to about $10 \mu \mathrm{g} / \mathrm{mL}$ GNR-757 and about $7 \mu \mathrm{g} / \mathrm{mL}$ of GNR-1064. At the same time, concentration of $\mathrm{NiSO}_{4}$ was $112 \mathrm{mg} / \mathrm{mL}$ or 11,000 greater than GNR-757 and 16,000 times greater than GNR-1064. Comparing effective concentrations of GNR and $\mathrm{NiSO}_{4}$ for similar OD has shown that the molar concentration of GNR is $1.5 \times 10^{8}$ smaller $(0.4 \mathrm{nM}$ and $0.6 \mathrm{M}$, respectively, see Fig. 9). The sensitivity of LOIS-3-D for GNR visualization in the tubes is about 440 fmole in the tube segment volume of $1.11 \mathrm{~mm}^{3}$. The tubes were illuminated with combined effective optical fluence of about $2.5 \mathrm{~mJ} / \mathrm{cm}^{2}$. This is at least eight times smaller than the maximum safe level of optical fluence permitted by American National Standards Institute $\left(20 \mathrm{~mJ} / \mathrm{cm}^{2}\right)$. This data demonstrated promising results for preclinical investigation of OCA 3-D high-sensitivity OA tomography by LOUIS 3-D with GNR as contrast agents for multiwavelength applications.

Multifunctional physiologically active HA can change properties not only of the stratum corneum, but also the dermis. ${ }^{49}$ It is known that removal of the stratum corneum dramatically increases skin permeability. ${ }^{74}$ However, removal of the full epidermis increases skin permeability by twice as much, and the epidermis can also offer a significant permeability barrier. $^{74}$ The swelling of scatterers in the dermis can lead light scattered 
in a more forward direction. ${ }^{73}$ Alternatively, hygroscopic OCAinduced dehydration may decrease the interfiber spacing, yielding more tightly packed fibers, which allows more constructive interference between scatterers, thereby decreasing the scattering coefficient and increasing light penetration as a result. ${ }^{73}$

Physical and chemical enhancement of transdermal transport of OCA was discussed in the literature. ${ }^{15}$ Ultrasound was used as a physical enhancer of OCA diffusivity, DMSO was used as a chemical enhancer, and glycerol combined with PEG-400 was used as the clearing agent. It was found that simultaneous application of ultrasound, DMSO, and OCA allowed one to reach the upper limit of skin OC. In our current report, we demonstrated that mixture of highly lipophilic PPG and hydrophilic PEG polymers with support by amphiphilic HA demonstrates an optimal base for coupling optical cleaner gel. Alternating lipophilic and hydrophilic layers of skin may absorb amphipathic compounds that possess both properties more effectively. Presented in this report are two steps; the order of OC with pretreatment of skin with HA before OCA application confirmed this mechanism.

$\mathrm{HA}$ as an enhancer for $\mathrm{OC}$ is unique because it is a biocompatible and naturally biodegradable molecular agent with amphiphilic properties. HA derivatives bearing both hydrophobic (indocyanine green) and hydrophilic (PEG) components were developed, but these substances did not possess the capability of enhancing penetration of light through skin. ${ }^{52}$ To transport drugs effectively through the cellular membrane and deliver them into the intracellular environment, several smart carrier systems based on HA composites were developed containing both synthetic or natural polymers. ${ }^{56} \mathrm{We}$ speculate that the method of HA pretreatment described in this work may be also effective for drug delivery. In recent years, HA has emerged as a likely candidate for intracellular delivery of various therapeutic and imaging agents because of its innate ability to recognize specific cellular receptors that are overexpressed on the membrane of abnormal cells. ${ }^{56}$ HA-based conjugates and hydrogels are made through conjugation of HA and functional group molecules. ${ }^{56}$ Hydrogels as HA-based $\mathrm{OC}$ composites for increased penetration of light require further investigation in the skin models because properties of HA and OCA may change when incorporated into a hydrogel. However, the potential role of HA in water solutions as an endogenous helper for efficient delivery OCA into skin and tissue is highly promising.

In summary, we proposed and tested a method for enhanced $\mathrm{OC}$ of skin and demonstrated its utility for OA imaging. Topical pretreatment of skin for about $30 \mathrm{~min}$ with $0.5 \% \mathrm{HA}$ in aqueous solution offers effective delivery of low molecular weight OCAs such as PPG, PEG, and their mixture. The tissue clearing effect was tested by measuring unscattered light transmission through treated tissue samples, and by detecting increased contrast in OA imaging of test targets through the treated skin. Our formulations for the OA coupling medium were recognized as being nontoxic, hypoallergenic, nonirritant, nonphototoxic, and pharmacologically inert, and they possess high chemical and physical stability.

\section{Acknowledgments}

Our research was sponsored in part by National Cancer Institute Grant No. R01CA167446 and in part by Seno Medical Instruments (San Antonio, Texas). The authors thank Paul Derry and Professor Eugene Zubarev from Rice University for their help with characterization of GNR.

\section{References}

1. E. A. Genina, A. N. Bashkatov, and V. V. Tuchin, "Glucose-induced optical clearing effects in tissues and blood," in Handbook of Optical Sensing of Glucose in Biological Fluids and Tissues, V. V. Tuchin, Ed., pp. 657-962, Taylor \& Francis, CRC Press, Boca Raton, Florida (2009).

2. E. A. Genina, A. N. Bashkatov, and V. V. Tuchin, "Tissue optical immersion clearing," Expert Rev. Med. Devices 7(6), 825-842 (2010).

3. D. Zhu et al., "Recent progress in tissue optical clearing," Laser Photonics Rev. 7(5), 732-757 (2013).

4. A. V. Liopo and A. A. Oraevsky, "Nanoparticles as contrast agents for optoacoustic imaging," in Nanotechnology for Biomedical Imaging and Diagnostics: From Nanoparticle Design to Clinical Applications, M. Berezin, Ed., pp. 111-149, John Wiley and Sons, New Jersey (2015).

5. V. P. Zharov et al., "In vivo high-speed imaging of individual cells in fast blood flow," J. Biomed. Opt. 11(5), 054034 (2006).

6. M. Sarimollaoglu et al., "Nonlinear photoacoustic signal amplification from single targets in absorption background," Photoacoustics 2(1), 1-11 (2014).

7. Q. Zhao et al., "Concentration dependence of optical clearing on the enhancement of laser-scanning optical-resolution photoacoustic microscopy imaging," J. Biomed. Opt. 19(3), 036019 (2014).

8. Z. Deng et al., "Viscous optical clearing agent for in vivo optical imaging," J. Biomed. Opt. 19(7), 076019 (2014).

9. B. W. Barry and A. C. Williams, "Permeation enhancement through skin," in Encyclopedia of Pharmaceutical Technology, J. Swarbrick and J. C. Boylan, Eds., pp. 449-493, Marcel Dekker, New York (1995).

10. D. W. Osborne and J. J. Henke, "Skin penetration enhancers cited in the technical literature," Pharm. Technol. 21, 58-66 (1997).

11. B. W. Barry, "Novel mechanisms and devices to enable successful transdermal drug delivery," Eur. J. Pharm. Sci. 14(2), 101-114 (2001).

12. A. E. Benson, "Transdermal drug delivery: penetration enhancement techniques," Curr. Drug Delivery 2(1), 23-33 (2005).

13. S. Golla et al., "Virtual design of chemical penetration enhancers for transdermal drug delivery," Chem. Biol. Drug Des. 79(4), 478-487 (2012).

14. Y. A. Menyaev et al., "Optical clearing in photoacoustic flow cytometry," Biomed. Opt. Express 4(12), 3030-3041 (2013).

15. E. A. Genina et al., "Optical coherence tomography monitoring of enhanced skin optical clearing in rats in vivo," J. Biomed. Opt. 19(2), 021109 (2014).

16. X. Yang et al., "Dynamic monitoring of optical clearing of skin using photoacoustic microscopy and ultrasonography," Opt. Express 22(1), 1094-1104 (2014).

17. O. Nadiarnykh and P. Campagnola, "SHG and optical clearing," in Second Harmonic Generation Imaging, pp. 169-189, CRC Press, Taylor \& Francis Group, Boca Raton, London, New York (2014).

18. V. Tuchin et al., "Light propagation in tissues with controlled optical properties," J. Biomed. Opt. 2(4), 401-417 (1997).

19. G. Vargas et al., "Use of an agent to reduce scattering in skin," Lasers Surg. Med. 24(2), 133-141 (1999).

20. V. V. Tuchin et al., "Optical clearing of skin using flashlamp-induced enhancement of epidermal permeability," Lasers Surg. Med. 38(9), 824-836 (2006)

21. V. V. Tuchin, "A clear vision for laser diagnostics," IEEE J. Sel. Top. Quantum Electron. 13(6), 1621-1628 (2007).

22. L. Oliveira et al., "Rat muscle opacity decrease due to the osmosis of a simple mixture," J. Biomed. Opt. 15(5), 055004 (2010).

23. R. Cicchi et al., "Contrast and depth enhancement in two-photon microscopy of human skin ex vivo by use of optical clearing agents," Opt. Express 13(7), 2337-2344 (2005).

24. X. Wen et al., "Controlling the scattering of intralipid by using optical clearing agents," Phys. Med. Biol. 54, 6917-6930 (2009).

25. K. V. Larin et al., "Optical clearing for OCT image enhancement and indepth monitoring of molecular diffusion," IEEE J. Sel. Top. Quantum Electron. 18(3), 1244-1259 (2012).

26. J. Wang et al., "Review: tissue optical clearing window for blood flow monitoring," IEEE J. Sel. Top. Quantum Electron. 20(2), 6801112 (2014).

27. V. V. Tuchin, "Tissue optics: light scattering methods and instruments for medical diagnostics," Vol. PM166, pp. 988, SPIE Press, Bellingham, Washington (2015). 
28. L. M. Oliveira et al., "Diffusion characteristics of ethylene glycol in skeletal muscle," J. Biomed. Opt. 20(5), 051019 (2015).

29. M. Khan et al., "Optical clearing of in vivo human skin: implications for light-based diagnostic imaging and therapeutics," Lasers Surg. Med. 34(2), 83-85 (2004).

30. V. V. Tuchin, "Tissue optics and photonics: biological tissue structures," J. Biomed. Photonics Eng. 1(1), 3-21 (2015).

31. Y. Grams and J. Bouwstra, "Penetration and distribution in human skin focusing on the hair follicle," in Percutaneous Absorption Drugs, Cosmetics, Mechanisms, Methods, R. L. Bronaugh and H. I. Maibach, Eds., pp. 177-191, Taylor \& Francis, CRC Press, Boca Raton, Florida (2005).

32. J. Hadgraft, "Modulation of the barrier function of the skin," Skin Pharmacol. Appl. Skin Physiol. 14(1), 72-81 (2001).

33. M. Khan et al., "Can topically applied optical clearing agents increase the epidermal damage threshold and enhance therapeutic efficacy?" Lasers Surg. Med. 35(2), 93-95 (2004).

34. M. K. Cowman and S. Matsuoka, "Experimental approaches to hyaluronan structure," Carbohydr. Res. 340(5), 791-809 (2005).

35. B. P. Toole, T. N. Wight, and M. I. Tammi, "Hyaluronan-cell interactions in cancer and vascular disease," J. Biol. Chem. 277(7), 4593-4596 (2002).

36. E. A. Turley, P. W. Noble, and L. Y. Bourguignon, "Signaling properties of hyaluronan receptors," J. Biol. Chem. 277(7), 4589-4592 (2002).

37. J. Necas et al., "Hyaluronic acid (hyaluronan): a review," Vet. Med. 53(8), 397-411 (2008).

38. K. Jansen et al., "A hyaluronan-based nerve guide: in vitro cytotoxicity, subcutaneous tissue reactions, and degradation in the rat," Biomaterials 25(3), 483-489 (2004).

39. L. P. Amarnath, A. Srinivas, and A. Ramamurthi, "In vitro hemocompatibility testing of UV-modified hyaluronan hydrogels," Biomaterials 27(8), 1416-1424 (2006).

40. R. Barbucci et al., "Hyaluronic acid hydrogel in the treatment of osteoarthritis," Biomaterials 23, 4503-4513 (2002).

41. I. Uthman, J. P. Raynauld, and B. Haraoui, "Intra-articular therapy in osteoarthritis," Postgrad. Med. J. 79(934), 449-453 (2003).

42. M. B. Brown and S. A. Jones, "Hyaluronic acid: a unique topical vehicle for the localized delivery of drugs to the skin," J. Eur. Acad. Dermatol. Venereol. 19(3), 308-318 (2005).

43. J. M. Medina, A. Thomas, and C. R. Denegar, "Knee osteoarthritis: should your patient opt for hyaluronic acid injection?" J. Fam. Pract. 55(8), 669-675 (2006).

44. J. Drobnik, "Hyaluronan in drug delivery," Adv. Drug Delivery Rev. 7(2), 295-308 (1991).

45. K. Jarvinen, T. Jarvinen, and A. Urtti, "Ocular absorption following topical delivery," Adv. Drug Delivery Rev. 16(1), 3-19 (1995).

46. S. T. Lim et al., "In vivo evaluation of novel hyaluronan/chitosan microparticulate delivery systems for the nasal delivery of gentamicin in rabbits," Int. J. Pharm. 231(1), 73-82 (2002).

47. W. Manuskiatti and H. I. Maibach, "Hyaluronic acid and skin: wound healing and aging," Int. J. Dermatol. 35(8), 539-544 (1996).

48. H. Trommer et al., "The effects of hyaluronan and its fragments on lipid models exposed to UV irradiation," Int. J. Pharm. 254(2), 223-234 (2003).

49. T. Brown, D. Alcorn, and J. Fraser, "Absorption of hyaluronan applied to the surface of intact skin," J. Invest. Dermatol. 113(5), 740-746 (1999).

50. L. Zhang et al., "Activatable hyaluronic acid nanoparticle as a theranostic agent for optical/photoacoustic image-guided photothermal therapy," ACS Nano 8(12), 12250-12258 (2014).

51. Z. Khatun et al., "A hyaluronic acid nanogel for photo-chemo theranostics of lung cancer with simultaneous light-responsive controlled release of doxorubicin," Nanoscale 7(24), 10680-10689 (2015).

52. K. Miki et al., "Near-infrared dye-conjugated amphiphilic hyaluronic acid derivatives as a dual contrast agent for in vivo optical and photoacoustic tumor imaging," Biomacromolecules 16(1), 219-227 (2015).

53. J. Wang, R. Shi, and D. Zhu, "Switchable skin window induced by optical clearing method for dermal blood flow imaging," J. Biomed. Opt. 18(6), 061209 (2013).

54. W. Wang, A. Cameron, and S. Ke, "Developing fluorescent hyaluronan analogs for hyaluronan studies," Molecules 17(2), 1520-1534 (2012).

55. M. Veiseh et al., "Imaging of homeostatic, neoplastic, and injured tissues by HA-based probes," Biomacromolecules 13(1), 12-22 (2012).
56. K. Choi et al., "Hyaluronic acid-based nanocarriers for intracellular targeting: interfacial interactions with proteins in cancer," Colloids Surf. B 99, 82-94 (2012).

57. R. Jin et al., "Synthesis and characterization of hyaluronic acid-poly (ethylene glycol) hydrogels via Michael addition: an injectable biomaterial for cartilage repair," Acta Biomater. 6(6), 1968-1977 (2009).

58. K. K. Vangara, J. L. Liu, and S. Palakurthi, "Hyaluronic acid-decorated PLGA-PEG nanoparticles for targeted delivery of SN-38 to ovarian cancer," Anticancer Res. 33(6), 2425-2434 (2013).

59. X. Xu et al., "Seedless synthesis of high aspect ratio gold nanorods with high yield," J. Mater. Chem. A 2, 3528-3535 (2014).

60. A. V. Liopo et al., "Seedless synthesis of gold nanorods using dopamine as a reducing agent," RSC Adv. 5, 91587-91593 (2015).

61. A. Liopo et al., "Highly purified biocompatible gold nanorods for contrasted optoacoustic imaging of small animal models," Nanosci. Nanotechnol. Lett. 4(7) 681-686 (2012).

62. R. Su et al., "Three-dimensional optoacoustic imaging as a new noninvasive technique to study long-term biodistribution of optical contrast agents in small animal models," J. Biomed. Opt. 17(10), 101506 (2012).

63. R. Su et al., "Laser opto acoustic tomography: towards new technology for biomedical diagnostics," Nucl. Instrum. Methods Phys. Res. A 720, 58-61 (2013).

64. D. A. Tsyboulski et al., "Enabling in vivo measurements of nanoparticles concentrations with three-dimensional optoacoustic tomography," J. Biophotonics 7(8), 581-588 (2014).

65. A. V. Liopo, R. Su, and A. A. Oraevsky, "Melanin nanoparticles as a novel contrast agent for optoacoustic tomography," Photoacoustics 3(1), 35-43 (2015).

66. K. Wang et al., "An imaging model incorporating ultrasonic transducer properties for three-dimensional optoacoustic tomography," IEEE Trans. Med. Imaging 30(2), 203-214 (2011).

67. M. A. Anastasio et al., "Half-time image reconstruction in thermoacoustic tomography," IEEE Trans. Med. Imaging 24(2), 199-210 (2005).

68. A. Fedorov et al., "3D slicer as an image computing platform for the quantitative imaging network," Magn. Reson. Imaging 30(9), 1323-1341 (2012).

69. G. Pitarresi et al., "Self-assembled amphiphilic hyaluronic acid graft copolymers for targeted release of antitumoral drug," J. Drug Target 18(4), 264-276 (2010).

70. G. M. Son et al., "Self-assembled polymeric micelles based on hyaluronic acid-g-poly(D,L-lactide-co-glycolide) copolymer for tumor targeting," Int. J. Mol. Sci. 15(9), 16057-16068 (2014).

71. M. C. Hacker and H. A. Nawaz, "Multi-functional macromers for hydrogel design in biomedical engineering," Int. J. Mol. Sci. 16(11), 27677-27706 (2015).

72. R. Wang et al., "Concurrent enhancement of imaging depth and contrast for optical coherence tomography by hyperosmotic agents," J. Opt. Soc. Am. B 18(7), 948-953 (2001).

73. X. Wen et al., "Enhanced optical clearing of skin in vivo and optical coherence tomography in-depth imaging," J. Biomed. Opt. 17(6), 066022 (2012).

74. S. N. Andrews, E. Jeong, and M. R. Prausnitz, "Transdermal delivery of molecules is limited by full epidermis, not just stratum corneum," Pharm. Res. 30(4), 1099-1109 (2013).

Anton Liopo is a lead scientist at TomoWave Laboratories Inc. (Houston, Texas) over the nanobiotechnology programs. He has created several nano-based contrast agents for optoacoustic (OA) imaging and sensing, laser nanothermolysis, molecular sensing, and methods of drug delivery. As visiting scientist, he has joined Rice University, Department of Chemistry, where he works on biomedical applications of nanocomposites. He has more than 70 publications in peer-reviewed journals, proceedings of international conferences, books, and two book chapters.

Richard Su graduated from the University of Texas in Austin with a Bachelor of Science in biomedical engineering in 2007 and is currently pursuing his $\mathrm{PhD}$ at the University of Houston. Over the last nine years in Fairway Medical Technologies and TomoWave Laboratories, he has been involved in a variety of OA tasks such as phantom design, in vitro and in vivo imaging, 2-D real-time and 3-D tomography imaging, prototype design, and system characterization and testing. 
Dmitri A. Tsyboulski obtained his $\mathrm{PhD}$ in chemistry from Rice University, Houston, Texas, USA, in 2006. He worked as a senior scientist at TomoWave Laboratories, Inc. (Houston, Texas, USA). His research expertise includes design of spectroscopic and imaging systems, nanoparticle characterization, and molecular spectroscopy. Currently, he is working as a senior research engineer at the Allen Institute for Brain Science.
Alexander A. Oraevsky presently leads TomoWave Laboratories as Chief Executive Officer and Chief Technology Officer and holds an adjunct professor position at the University of Houston. He is a pioneer in the field of biomedical optoacoustics and has published eight book chapters and over 200 scientific papers. He is the primary inventor of 21 patents and the recipient of multiple research awards, including the Berthold Leibinger Innovations Prize. 